\title{
Os comportamentos de risco para os transtornos alimentares atenuam a agilidade e a impulsão vertical em atletas de esportes de combate?
}

http://dx.doi.org/10.11606/1807-5509201700040721

\author{
Leonardo de Sousa FORTES* \\ Saulo Fernandes Melo de OLIVEIRA* \\ Lilyan Carla Vaz MENDONÇA* \\ Geraldo José Santos OLIVEIRA* \\ Pedro Pinheiro PAES* \\ António Manuel Leal Ferreira Mendonça da FONSECA**
}

*Departamento de Educação Física

e Desportos, Universidade Federal de Pernambuco, Recife, PE, Brasil.

**Faculdade

de Desportos,

Universidade do

Porto, Porto, Portugal

\section{Resumo}

Desconhece-se a relação entre desempenho físico e comportamentos de risco para os transtornos alimentares (CRTA). 0 objetivo foi comparar a agilidade e a impulsão vertical entre atletas de esportes de combate do sexo masculino com e sem risco para os transtornos alimentares. Participaram 113 atletas com idade entre 12 e 36 anos. 0 Eating Attitudes Test (EAT-26) foi utilizado para avaliar CRTA. Utilizou-se 0 teste vai-e-vem ( 5 metros) para avaliar agilidade. 0 teste de impulsão vertical foi utilizado para avaliar a potência anaeróbia de membros inferiores. Conduziu-se análise multivariada de covariância (MANOVA) para comparar os testes motores em função das classificações dicotômicas do EAT-26. Os resultados indicaram diferença estatisticamente significante da impulsão vertical entre atletas com (Média $=2,50 \mathrm{~m}$ ) e sem risco (Média $=2,58 \mathrm{~m})$ para os transtornos alimentares $\left(F_{(2,111)}=12,34 ; p=0,041\right)$. Os achados não apontaram diferenças para a agilidade entre atletas com (Média=20,57 segundos) e sem risco (Média=20,34 segundos) para os transtornos alimentares $\left(F_{(2,111)}=2,57 ; p=0,22\right)$. Concluiu-se que os comportamentos de risco para transtornos alimentares estiveram relacionados com o menor desempenho na impulsão vertical em atletas de esportes de combate do sexo masculino, fato não replicado para a agilidade.

Palavras-ChaVe: Desempenho Motor; Transtornos Alimentares; Atletas.

\section{Introdução}

Tratando-se de atletas, as sessóes de treinamento buscam maximizar o desempenho, seja técnico, tático e/ou físico ${ }^{1}$. Considerando o desempenho físico, pesquisadores têm apontado a agilidade e a potência anaeróbia como determinantes para se obter sucesso nos esportes de combate ${ }^{2}$. Segundo Ronnestad e Ellefsen ${ }^{3}$, o desempenho físico de atletas pode ser avaliado por meio de testes motores, tais como: potência e resistência anaeróbia (Teste de Wingate), velocidade (corrida), agilidade, impulsão vertical/horizontal, arremesso de "medicine ball", entre outros. Mais especificamente, tratando-se do judô, o Special Judo Fitness Test ${ }^{4}$ é bastante utilizado para avaliar a capacidade anaeróbia. Salienta-se que a avaliação do desempenho físico durante uma temporada competitiva é importante para se monitorar o progresso dos atletas. No entanto, existem comportamentos que acarretam redução do desempenho físico e parece que os comportamentos de risco para os transtornos alimentares podem ser um destes 5 .

A restrição alimentar por longos períodos, os métodos purgativos (laxantes, diuréticos, autoindução de vômito e inibidores de apetite) e a compulsão alimentar são considerados comportamentos de risco para os transtornos alimentares ${ }^{5}$. Estudos indicam que cerca de $20 \%$ dos atletas do sexo masculino são acometidos pelos comportamentos de risco para os transtornos alimentares ${ }^{6,7}$. Mais especificamente, os atletas dos esportes de combate parecem utilizar estes 
comportamentos com mais frequência. De acordo com Rouverx et al. ${ }^{8}$, aproximadamente $50 \%$ dos atletas de esportes de combate utilizam métodos purgativos para diminuir a massa corporal. Mais agravante ainda, estudos revelam que cerca de $70 \%$ dos atletas de esportes de combate adotam os métodos de rápida redução de massa corporal em dias antecedentes a competição-alvo ${ }^{9,10}$.

Os esportes de combate [boxe, jiu-jitsu, judô, mixed martial arts (MMA) muay thai, taekwondo, entre outros] são modalidades individuais, nas quais o objetivo é superar o adversário ${ }^{11}$. Geralmente um combate é encerrado após uma ação explosiva (potência anaeróbia) executada com sucesso ${ }^{12}$. Salienta-se, todavia, que o tempo médio de duração das lutas é de 5 minutos ${ }^{13}$. Logo, o principal substrato energético utilizado durante os combates é o glicogênio muscular ${ }^{14}$. Ademais, uma das peculiaridades dos esportes de combate é a divisão das categorias por massa corporal. Cabe destacar que tanto atletas quanto treinadores associam a diminuição da massa corporal com a maximização do desempenho físico ${ }^{15}$. Neste sentido, os atletas dos esportes de combate podem adotar os comportamentos de risco para os transtornos alimentares com a premissa de reduzir a massa corporal.

Nos esportes de combate, por inúmeras vezes ao longo do ano, o atleta é recomendado pelo seu treinador a competir em categorias abaixo de sua massa corporal considerada normal para a sua estatura. Sendo assim, muitos competidores usufruem de métodos não saudáveis para reduzir rapidamente a massa corporal em dias antecedentes à competição principal, acreditando que essas atitudes sejam vantajosas sobre os seus oponentes. Neste sentido, a "ciclagem" de massa corporal costuma ser frequente nas carreiras esportivas destes atletas. Todavia, muitos treinadores não demonstram conhecimento relacionado à rápida redução de peso e as disfunçôes cardiovasculares, desequilíbrio hormonal, aumento do risco de lesões, diminuição

\section{Método}

\section{Consideraçóes éticas}

O estudo obteve aprovação do Comitê de Ética em Pesquisa Envolvendo Seres Humanos de conteúdo mineral ósseo e transtornos alimentares que esta pode ocasionar ${ }^{14}$.

Em adição, parece que os responsáveis pelas Federaçóes dos esportes de combate contribuem para o aumento da frequência de utilizaçáo de métodos para a rápida redução de massa corporal entre os atletas. Por exemplo, no judô e no jiujitsu é permitido o atleta se pesar várias vezes até atingir a massa corporal desejada para competir em determinada categoria. Permite-se, ainda, que o atleta vá para dentro de outra sala e realize exercícios extenuantes com o uso de agasalhos ou induza o vômito com o propósito de atenuar água corporal. Além disso, de acordo com Artioli et al. ${ }^{13}$, o tempo entre a pesagem oficial e o início da primeira luta em eventos competitivos de esportes de combate é em média de 4 horas, o que favorece a recuperação imediata da massa corporal reduzida por meio de reidratação e alimentação a base de carboidratos. Em síntese, todas estas concessóes possibilitam a utilização de métodos de rápida redução de massa corporal.

Bratland-SANdA E Sundgot-Borgen ${ }^{15}$ ressaltam que o desempenho físico de atletas pode ser afetado negativamente pelos comportamentos de risco para os transtornos alimentares, principalmente nos esportes de combate. Entretanto, necessita-se conduzir investigação científica para analisar se tal afirmação é verdadeira. Não se tem conhecimento de estudo que tenha buscado avaliar a relação entre os comportamentos de risco para os transtornos alimentares e o desempenho físico. Do ponto de vista prático, este tipo de pesquisa contribui para a área das ciências do esporte por investigar se a utilização de comportamentos patológicos para a redução de massa corporal (comportamento comum na cultura dos esportes de combate), de fato, atenua o desempenho físico de atletas. Diante do exposto, o presente estudo teve como objetivo comparar o desempenho de agilidade e impulsão vertical entre atletas de esportes de combate do sexo masculino com e sem risco para os transtornos alimentares.
(CAAE - 31312014.0.0000.5208). Os responsáveis, assim como os atletas, assinaram um Termo de Consentimento Livre e Esclarecido (TCLE), que explicava os objetivos e os procedimentos do estudo. 


\section{Participantes}

Para inclusão dos sujeitos na pesquisa, foram adotados alguns critérios, a saber: 1) Ter participado de Campeonatos no ano de 2014 e; 2) treinar no mínimo 6 horas por semana na sua respectiva modalidade esportiva (esporte de combate). A amostra do estudo constituiu-se de atletas do sexo masculino de esportes de combate (jiu-jitsu e judô), com idade igual ou superior a 12 anos, selecionados de forma não probabilística e por conveniência. Foram recrutados 137 atletas do sexo masculino. Entretanto, 24 atletas foram excluídos do estudo pelos seguintes motivos: não responderam os questionários na sua totalidade; não realizaram os testes motores ou; 3) não participaram das mediçóes antropométricas. Por fim, a pesquisa contou com 113 atletas do sexo masculino [jiu-jitsu ( $n=26)$ e judô $(n=87)]$, com média de 17,56 $( \pm 4,94)$ anos para a idade, $10,76( \pm 1,87)$ horas para o regime semanal de treino, 5,23 $( \pm 2,11)$ anos de experiência na sua respectiva modalidade esportiva e 19,21 $( \pm 6,87)$ para o percentual de gordura. Destaca-se, ainda, que $75,2 \%$ dos atletas competiam em nível nacional e os $24,8 \%$ restante competiam em nível internacional.

\section{Instrumentos}

\section{Eating attitudes test (EAT-26)}

O EAT-26 foi utilizado para avaliar comportamentos de risco para os transtornos alimentares. Consta de um questionário com 26 questôes, que busca avaliar a recusa patológica alimentar, os métodos purgativos (laxantes e diuréticos) e a influência do ambiente sobre a ingestão de alimentos. A versão utilizada foi a de BighetTi et al. ${ }^{16}$ Estes autores identificaram adequada consistência interna do instrumento $(\alpha=0,82)$. Para a presente amostra, identificou-se valor de 0,84 para a consistência interna, avaliada pelo alpha de Cronbach. A pontuação do EAT- 26 é feita pela soma dos itens. Escore igual ou maior que 21 indica susceptibilidade para os transtornos alimentares. Existem seis opçóes de respostas (sempre $=3$, muitas vezes $=2$, frequentemente $=1$, poucas vezes $=0$, quase nunca $=0$, nunca $=0$ ), no entanto, a questão 25 apresenta pontuação invertida (sempre $=0$, muitas vezes $=0$, frequentemente $=0$, poucas vezes $=1$, quase nunca $=2$, nunca $=3$ ).

\section{Agilidade $^{17}$}

Para a realização do teste foram colocados 2 cones com distância de $5 \mathrm{~m}$ entre eles e 1,2 m de largura entre os mesmos (FIGURA 1). Os avaliados percorreram 10 vezes de um cone ao outro ( $5 \mathrm{~m}$ de distância) na maior velocidade possível (com ultrapassagem dos cones). Cada participante teve apenas uma tentativa para a realização do teste. $\mathrm{O}$ tempo gasto pelo participante foi obtido por intermédio de um cronômetro. Salienta-se que o teste foi realizado em piso antiderrapante. Vale ressaltar que a escolha deste teste se deu em razão da fácil aplicabilidade e do pequeno espaço requerido para a sua realização.

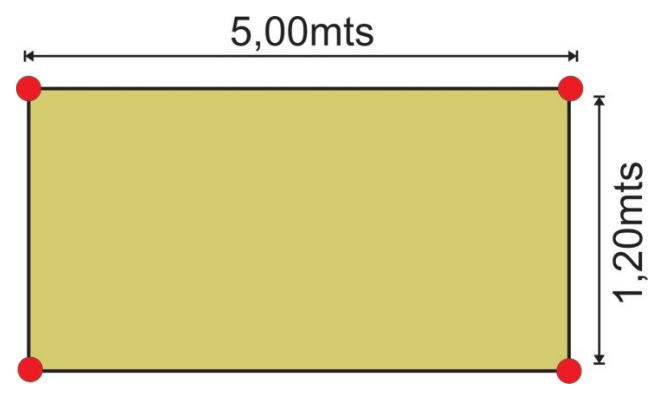

FIGURA 1 - Teste de agilidade: posicionamento dos cones.

\section{Impulsáo vertical ${ }^{18}$}

Para a realização do teste foi fixado uma fita métrica de 3 metros de comprimento em uma parede lisa, com precisão de $0,5 \mathrm{~cm}$. O avaliado foi posicionado lateralmente a fita métrica fixada na parede, com os pés totalmente apoiados no solo, o braço mais próximo da parede totalmente 
estendido acima da cabeça. Em seguida, o avaliado deveria flexionar os joelhos e realizar uma impulsão contra o movimento em esforço máximo. O braço estendido marcou o ponto mais alto alcançado pelo avaliado. Para melhor marcação foi utilizado pó de giz nos dedos do avaliado. Cabe ressaltar que a escolha deste teste ocorreu em função da fácil aplicabilidade e da rapidez na coleta dos dados. Salienta-se que a estatura dos atletas poderia influenciar nos resultados do teste. Logo, a estatura foi controlada estatisticamente.

\section{Antropometria}

Para a verificação das dobras cutâneas, foi utilizado um compasso (adipômetro) científico da marca LANGE ${ }^{\oplus}$ (Cambridge Scientific Industries Inc.), com precisão de $1 \mathrm{~mm}$. Os procedimentos para a coleta das dobras cutâneas foram realizados de acordo com as padronizaçóes determinadas pela International Society for Advancement for Kineanthropometry ${ }^{19}$. Realizou-se o cálculo do erro técnico de medida (ETM) proposto por SiLva et al. ${ }^{20}$, excluindo dados com variância maior que $10 \%$.

O percentual de gordura ( $\% \mathrm{G})$ foi estimado pelo método duplamente indireto. Foram mensuradas as dobras cutâneas triciptal e subescapular em atletas com idade inferior a 18 anos. Em atletas com idade igual ou superior a 18 anos foram mensuradas as dobras triciptal, peitoral e subescapular. Estas medidas foram tomadas de forma rotacional e coletadas três vezes, sendo considerada a média dos valores. Para o cálculo da densidade corporal foram utilizados os protocolos de SLAUGHTER et al. ${ }^{21}$ [considerou-se a etnia e a etapa maturacional em função da idade cronológica (púbere - 12 a 14 anos; e pós-púbere - 15 a 17 anos)] e JACKson e Pollock ${ }^{22}$, criados para adolescentes e adultos, respectivamente. $\mathrm{O} \% \mathrm{G}$ foi estimado mediante equação desenvolvida por SIRI ${ }^{23}$.

A massa corporal foi mensurada por uma balança digital portátil da marca Tanita com precisão de 0,1 $\mathrm{kg}$ e capacidade máxima de $220 \mathrm{~kg}$. Esta medida foi aferida utilizando os seguintes critérios: atleta em pé, descalço com o mínimo de roupas utilizadas na modalidade esportiva específica, posicionado no centro da plataforma com os braços estendidos ao longo do corpo e com olhar fixo à frente, de modo a evitar oscilação na leitura da medida.

Foi utilizado estadiômetro portátil com precisão de $0,1 \mathrm{~cm}$ e altura máxima de $2,20 \mathrm{~m}$ da marca
Tonelli para aferir a estatura dos atletas. Os indivíduos deveriam ficar em pé, olhando para o infinito, sem sapatos, com calcanhares, glúteos, dorso e cabeça encostados ao plano vertical e os pés e calcanhares unidos formando um ângulo de $45^{\circ}$. Nesta posição, a peça do estadiômetro, em ângulo reto, foi posicionada sobre o topo da cabeça do atleta e a medida foi anotada.

\section{Procedimentos}

Inicialmente, foi obtido autorização dos treinadores das equipes para a realização da coleta dos dados. Em seguida, os atletas foram abordados em seu local de treinamento, no qual foram informados sobre os procedimentos do estudo. Solicitou-se que os atletas menores de 18 anos levassem aos seus responsáveis o TCLE e o mesmo, assinado pelo seu responsável, deveria ser entregue na semana subsequente.

A pesquisa foi dividida em dois momentos. A primeira etapa foi destinada à aplicação do EAT-26, ao passo que o segundo momento foi destinado a aplicação dos testes motores e mediçóes antropométricas. Destaca-se que em razão de alguns treinadores pedirem rapidez nas coletas de dados, foram escolhidos testes motores de fácil aplicabilidade, baixa onerosidade e rápida duração.

Concernente ao EAT-26, todos os atletas receberam a mesma orientação verbal. Todas as dúvidas foram esclarecidas e não foram permitidas comunicaçôes entre os sujeitos. O preenchimento do questionário foi de forma voluntária e sem limite de tempo.

Realizou-se uma sessão de familiarização dos testes motores (agilidade e impulsão vertical) $72 \mathrm{~h}$ antes das coletas de dados com a premissa de evitar efeito de aprendizagem. Antes da realização dos testes motores foi realizado um aquecimento com duração de 3 minutos. Foram procedidos saltos verticais, breves "sprints" e deslocamento em baixa velocidade. Por conseguinte, foram realizadas as aferiçôes antropométricas.

\section{Analise estatística}

Utilizou-se o teste Kolmogorov Smirnov para avaliar a distribuição das variáveis da pesquisa. Em razão da não violação da normalidade dos dados, utilizaram-se técnicas paramétricas. Conduziu-se análise multivariada de covariância (MANOVA), controlando a idade, a estatura e o percentual de 
gordura para comparar os testes motores (impulsão vertical e agilidade) em função das classificações dicotômicas do EAT-26 (com e sem risco para os transtornos alimentares). O tamanho do efeito de Cohen, representado pela letra "d", foi calculado para indicar diferenças do ponto de vista prático. O critério de Thalheimer e $\operatorname{CoOK}^{24}$ foi utilizado para classificar o tamanho do efeito (baixo, médio e alto). Os dados foram tratados no software SPSS 21.0, adotando-se nível de significância de 5\%.

\section{Resultados}

Os achados indicaram que $36,2 \%$ dos atletas demonstraram risco para o desencadeamento de transtornos alimentares (EAT-26 $\geq 21$ ). Mais especificamente, os resultados revelaram que $42,3 \% \mathrm{e}$
$34,5 \%$ dos atletas de jiu-jitsu e judô, respectivamente, indicaram susceptibilidade para desencadear transtorno alimentar. Os dados descritivos das variáveis do estudo estão apresentados na TABELA 1.

TABELA 1 - Dados descritivos (mínimo, máximo, média e desvio padrão) das variáveis da investigação.

\begin{tabular}{lcc}
\hline \multicolumn{1}{c}{ Variável } & Média $( \pm \mathrm{DP})$ & IC $(\mathbf{9 5 \%})$ \\
\hline EAT-26 & $21,02( \pm 18,79)$ & $11,22-26,78$ \\
\hline Agilidade (segundos) & $20,44( \pm 1,92)$ & $16,33-23,03$ \\
\hline Impulsão vertical $(\mathrm{m})$ & $2,55( \pm 0,17)$ & $2,19-2,82$ \\
\hline \% Gordura & $19,21( \pm 6,87)$ & $11,06-29,75$ \\
\hline Idade (anos) & $17,56( \pm 4,94)$ & $12,86-32,03$ \\
\hline Regime de treino semanal (horas) & $10,76( \pm 1,87$ & $8,50-15,63$ \\
\hline Experiência no esporte (anos) & $5,23( \pm 2,11)$ & $3,19-9,44$ \\
\hline
\end{tabular}

EAT-26: Eating Attitudes Test; \% Gordura: Percentual de Gordura; DP: Desvio Padrão; IC: Intervalo de Confiança
Os resultados da MANOVA (TABELA 2) indicaram diferença estatisticamente significante da impulsão vertical entre atletas com e sem risco para os transtornos alimentares $\left(F_{(2,111)}=12,38\right.$; $p=0,041)$, embora o tamanho do efeito de Cohen tenha sido baixo $(d=0,4)$. Em contrapartida, os achados não apontaram diferenças para a agilidade entre atletas com e sem risco para os transtornos alimentares $\left(F_{(2,111)}=2,57 ; p=0,22\right.$; $d=0,1)$. Salienta-se que a idade demonstrou colinearidade com os escores do EAT-26 $\left(F_{(2,111)}=7,45 ; p=0,03\right)$, fato não revelado para o percentual de gordura $\left(F_{(2,111)}=1,94 ; p=0,30\right)$ nem para a estatura $\left(F_{(2,11)}=1,76 ; p=0,26\right)$.

TABELA 2 - Média e erro padrão das variáveis motoras (impulsão vertical e agilidade) em razão das classificações dicotômicas do EAT-26.

\begin{tabular}{|c|c|c|}
\hline Variável & $\begin{array}{c}\text { Sem risco }(\text { EAT-26<21) } \\
(n=72)\end{array}$ & $\begin{array}{c}\text { Com risco }(\text { EAT-26 } \geq 21) \\
(n=41)\end{array}$ \\
\hline Impulsão vertical (m) & $2,58(0,16)^{a}$ & $2,50(0,21)$ \\
\hline Agilidade (segundos) & $20,34(0,23)$ & $20,57(0,30)$ \\
\hline
\end{tabular}

EAT-26: Eating Attitudes Test; ap $<0,05$ em relação ao grupo "com risco".

\section{Discussão}

O objetivo do presente estudo foi comparar o desempenho de agilidade e impulsão vertical entre atletas de esportes de combate do sexo masculino com e sem risco para os transtornos alimentares. 
Os resultados apontaram diferença significativa da impulsão vertical entre os atletas com e sem riscos para os transtornos alimentares. Todavia, não foi identificada diferença significante para a agilidade.

Os achados do presente estudo indicaram prevalência de $34,5 \%$ de comportamentos de risco para transtornos alimentares em atletas de judô, corroborando a literatura científica. Por exemplo, Vieira et al. ${ }^{25}$ avaliaram atletas adultos de Judô e verificaram prevalência de 33\%. Do mesmo modo, Coufalová et al. ${ }^{26}$ avaliaram atletas de diversos esportes de combate e encontraram prevalência de aproximadamente $43 \%$ para os comportamentos de risco para os transtornos alimentares, o que está de acordo com os achados da presente investigação para os atletas de jiu-jitsu. Ressalta-se, todavia, que os métodos para a redução de massa corporal (indução de vômito, restrição alimentar e uso de fármacos) podem acarretar diminuiçáo da força muscular e potência anaeróbia de atletas ${ }^{5}$.

As Federaçóes dividem os esportes de combate em categorias de massa corporal no sentido de se tentar equilibrar as valências motoras dos atletas como força, potência e agilidade ${ }^{13}$, embora a massa corporal possua baixa relaçáo com tais variáveis motoras ${ }^{2}$. No entanto, parece que os atletas de esportes de combate costumam utilizar os comportamentos de risco para os transtornos alimentares com a premissa de atenuar a massa corporal para competir em categoria abaixo ${ }^{8}$. Destaca-se, sobretudo, que tais comportamentos podem ter efeitos negativos no desempenho físico, por exemplo, a diminuição da potência anaeróbia 5 . Neste sentido, considerando a impulsão vertical, os resultados do presente estudo corroboram esse pressuposto. Os atletas com escores iguais ou superiores a 21 no EAT-26 demonstraram menor desempenho no teste de impulsão vertical em comparação aos atletas que não indicaram risco para os transtornos alimentares (EAT-26 < 21). Segundo Brito et al. ${ }^{2}$, os atletas que utilizam métodos de rápida redução de massa corporal (laxantes, diuréticos, sauna e inibidores de apetite) podem ter algumas valências motoras de orientação anaeróbia deterioradas, o que, de certo modo, explica os achados da presente investigação a respeito da impulsão vertical.

Em contrapartida, referindo-se à agilidade, o presente estudo não indicou nenhuma diferença significante entre atletas com e sem risco para os transtornos alimentares. Considerando o tempo médio de duração do teste de agilidade para os atletas do presente estudo (20,44 segundos), é importante salientar que o principal sistema energético utilizado para este teste foi o glicogênio muscular. Embora pesquisadores ressaltem que os métodos patológicos para a redução de massa corporal podem gerar redução dos estoques de glicogênio muscular ${ }^{15}$, é importante destacar que o desempenho em exercícios de curta duração (<30 segundos) pode náo ser afetado pelo baixo estoque de glicogênio muscular ${ }^{14}$. Logo, mesmo com baixo estoque de glicogênio muscular, o atleta pode ter um bom desempenho em atividades com duração inferior a 30 segundos, desde que não sejam realizados esforços repetidos, o que pode justificar os achados concernentes a agilidade.

Embora o presente estudo tenha verificado achados inéditos para a literatura científica, o mesmo apresentou limitaçóes. Uma delas foi utilizar questionário como instrumento de avaliação, podendo o resultado náo refletir a realidade devido as respostas com caráter subjetivo ${ }^{27-29}$. Outra limitação foi o método duplamente indireto para avaliar o \%G. Ademais, a mensuração de dados antropométricos foi conduzida após a realização dos testes motores, o que pode ter subestimado as medidas. Destaca-se também a utilização de cronômetro para avaliar o tempo no teste de agilidade, bem como a utilização de fita métrica e pó de giz para mensurar a impulsão vertical. Em adição, destaca-se que as açóes musculares realizadas nos testes motores não são específicas dos esportes de combate. Logo, os achados da presente investigação devem ser analisados com cautela. Todavia, este estudo, mesmo com as suas limitações mostrou importantes resultados sobre as consequências que os comportamentos de riscos para os transtornos alimentares podem exercer sobre o desempenho físico de atletas de esportes de combate.

Por fim, concluiu-se que os comportamentos de risco para os transtornos alimentares estiveram relacionados com o menor desempenho na impulsão vertical em atletas de esportes de combate do sexo masculino, fato náo replicado para a agilidade. Entretanto, faz-se necessário a condução de estudos com delineamento longitudinal que busquem avaliar a influência dos comportamentos de risco para os transtornos alimentares sobre o desempenho físico de atletas. 


\section{Abstract}

Does disordered eating decrease the agility and vertical jump in combat sports athletes?

It is unknown the relationship between anaerobic performance and disordered eating (DE). The objective was to compare agility and vertical jump among male combat sports athletes with and without risk for eating disorders. 113 athletes aged between 12 and 36 years were participants. The Eating Attitudes Test (EAT-26) was used to assess DE. We used the comes-and-back test (5 meters) to evaluate agility. The vertical jump test was used to evaluate the lower limbs anaerobic power. It conducted a multivariate analysis of covariance (MANOVA) to compare the motor tests according to dichotomous ratings of EAT-26. The results indicated a statistically significant difference the vertical jump between athletes with (Average $=2,50 \mathrm{~m}$ ) and without (Average $=2,58 \mathrm{~m})$ risk for eating disorders $\left(F_{(2,111)}=12.34 ; p=0.041\right)$. The findings showed no differences for agility/speed between athletes with (Average $=20,57$ seconds) and without (Average $=20,34$ seconds) risk for eating disorders $\left(F_{(2,111)}=2.57 ; p=0.22\right)$. It was concluded that the $D E$ are associated with attenuation of vertical jump in male combat sports athletes, which was not replicated to the agility.

KeYwords: Physical Performance; Eating Disorders; Athletes.

\section{Agradecimentos}

Aos atletas pela colaboração em participar da investigação. Ao Banco Santander pelo financiamento de parte do projeto.

\section{Referências}

1. Sundgot-Borgen J, Torstveit MK. Aspects of disordered eating continuum in elite high-intensity sports. Scand J Med Sci Sports. 2010;20(supl 2):112-21.

2. Brito CS, Roas AFM, Brito ISS, Marins JCB, Cordova C, Franchini E. Methods of body-mass reduction by combat sport athletes. Int J Sport Nutr Exercise Metabolism. 2012;22(1):89-97.

3. Ronnestad BR, Ellefsen HS. Block periodization of high-intensity aerobic intervals provides superior training effects in trained cyclists. Scan J Med Sci Sports. 2014;24(1):34-42.

4. Detanico D, Santos SG. Specific evaluation in judo: a review of methods. Rev Bras Cineantropom Desempenho Hum. 2012;14(6):738-48.

5. El Ghoch M, Soave F, Cafugi S, Grave D. Eating disorders, physical fitness and sport performance: a systematic review. Nutrients. 2013;5(5):5140-60.

6. Fortes LS, Ferreira MEC, Oliveira SMF, Cyrino ES, Almeida SS. A socio-sports model of disordered eating among Brazilian male athletes. Appetite. 2015;92:29-35.

7. Petrie T, Galli N, Greenleaf C, Reel J, Carter J. Psychological correlates of bulimic-symptomatology among male athletes. Psychol Sport Exercise. 2014;15(6):680-87.

8. Rouveix M, Bouget M, Pannafieux C, Champely S, Filaire E. Eating attitudes, body esteem, perfectionism and anxiety of judo athletes and nonathletes. Int J Sports Med. 2007;28(2):340-45.

9. Janiszewska K, Przybyłowicz KE. Pre-competition weight loss among Polish taekwondo competitors: occurrence, methods and health consequences. Arch Budo. 2015;11(1):41-5.

10. Durguerian A, Bougard C, Drogou C, Sauvet F, Chennaoui M, Filaire E. Weight loss, performance and psychological related states in high-level weightlifters. Int J Sports Med. 2015;25(2):110-8.

11. Filaire E, Rouveix M, Pannafieux C, Ferrand C. Eating attitudes, perfectionism and body-esteem of elite male judoists and cyclists. J Sports Sci Med. 2007;6(1):50-7.

12. Artioli GG, Scagliusi FB, Polacow VO, Gualano B, Lancha Junior AH. Magnitude e métodos de perda rápida de peso em judocas de elite. Rev Nutr. 2007;20(3):307-15. 
13. Artioli GG, Franchini E, Nicastro H, Sterkwicz S, Solis MY, Lancha Junior AH. The need of a weight management control program in judo: a proposal based on the successful case of wrestling. J Int Society Sport Nutr. 2010;15(7):1-5.

14. Lima-Silva AE, Fernandes TC, Oliveira FR, Nakamura FY, Gevaerd MS. Metabolismo do glicogênio muscular durante o exercício físico: mecanismos de regulação. Rev Nutr. 2007;20(4):417-29.

15. Bratland-Sanda S, Sundgot-Borgen J. Eating disorders in athletes: overview of prevalence risk factors and recommendations for prevention and treatment. Eur J Sport Sci. 2012;1(1):1-10.

16. Bighetti F, Santos CB, Santos JE, Ribeiro RPP. Tradução e avaliação do eating attitudes test em adolescentes do sexo feminino de Ribeirão Preto, São Paulo. J Bras Psiquiatr. 2004;53(6):33946.

17. Vaz L, Vasilica I, Carreras, D, Kraak W, Nakamura FY. Physical fitness profiles of elite under-19 rugby union players. J Sports Med Physical Fitness. 2016;56(4):415-21.

18. Freitas AS, Figueiredo AJB, Freitas ALR, Rodrigues VD, Cunha AC, Deusdará FF, et al. Biological maturation, body morphology and physical performance in 8-16 year-old obese girls from Montes Claros - MG. J Hum Kinects. 2014 set;43:169-76.

19. The International Society for Advancement for Kineanthropometry [homepage na Internet]. Australia: National Library of Australia [citado em 2018 mar 9]. Available from: <https://goo.gl/k4W6x4>

20. Silva DAS, Pelegrini A, Pires-Neto CS, Vieira MFS, Petroski EL. O antropometrista na busca de dados mais confiáveis. Rev Bras Cineantr Desemp Hum. 2011;13(1):82-5.

21. Slaughter MH, Lohman TG, Boileau R, Hoswill CA, Stillman RJ, Yanloan MD. Skinfold equations for estimation of body fatness in children and youth. Human Biology. 1988;60(3):709-23.

22. Jackson AS, Pollock ML. Generalized equations for predicting body density of men. Br J Nutr. 1978;40(3):497-504.

23. Siri WE. The gross composition of the body. In: Tobias CA, Lawrence JH, editores. Advances in biological and medical physics. New York: Academic Press; 1956. p. 239-80.

24. Thalheimer W, Cook S. How to calculate effect sizes from published research articles: a simplified methodology. Somerville: Work-Learning Research; 2002.

25. Vieira JLL, Oliveira LP, Vieira LF, Vissoci JRN, Hoshino EF, Fernandes SL. Distúrbios de atitudes alimentares e sua relação com a distorção da auto-imagem corporal em atletas de judô do estado do Paraná. Rev Educaçáo Física/UEM. 2006;17(2):177-84.

26. Coufalová K, Prokešová E, Malý T, Heller J. Body weight reduction in combat sports. Arch Budo. 2013;9(4):267-72.

27. Fortes LS, Neves CM, Filgueiras JF, Almeida SS, Ferreira MEC. Body dissatisfaction, psychological commitment to exercise and eating behavior in young athletes from aesthetic sports. Rev Bras Cineantr Desemp Hum. 2013;15(6):695-704.

28. Fortes LS, Ferreira MEC. Comparison of body dissatisfaction and inappropriate eating behavior in adolescent athletes of different sports. Rev Bras Educ Fís Esporte. 2011;25(4):707-16.

29. Fortes LS, Matta MO, Paes ST, Ferreira MEC. Fatores de risco associados ao comportamento alimentar inadequado em futebolistas. Rev Bras Educação Fís Esporte. 2012;26(3):447-54.

\begin{tabular}{|c|c|}
\hline $\begin{array}{r}\text { ENDEREÇO } \\
\text { Leonardo de Sousa Fortes } \\
\text { Rua Clóvis Beviláqua, 163/1.003 - Madalena } \\
50710-330-\text { Recife }-\mathrm{PE} \mathrm{-} \mathrm{BRASIL} \\
\text { e-mail: leodesousafortes@hotmail.com; leonardo. } \\
\text { fortes@ufpe.br }\end{array}$ & $\begin{array}{l}\text { Recebido para publicação: 29/o9/2015 } \\
\text { Revisado: 29/12/2015 } \\
\text { Aceito: 26/02/2016 }\end{array}$ \\
\hline
\end{tabular}

